\title{
Allergisches Kontaktekzem nach Anwendung eines grünen Permanentmarkers
}

\section{Contact Allergy Following Use of a Green Permanent Marker}

\author{
Autoren \\ Felix Wagner, Jasmin Woitalla-Bruning \\ Institut \\ Zentrum für Venen- und Dermatochirurgie, Krankenhaus \\ Tabea GmbH \& Co. KG \\ Schlüsselwörter \\ Kontaktekzem, Edding, allergisches Kontaktekzem Typ IV, \\ grüner Permanentmarker \\ Key words \\ contact allergy, green permanent marker \\ Bibliografie \\ Phlebologie 2020; 49: 279-282 \\ DOI 10.1055/a-1171-4952 \\ ISSN 0939-978X \\ (c) 2020. Thieme. All rights reserved. \\ Georg Thieme Verlag KG, Rüdigerstraße 14, \\ 70469 Stuttgart, Germany \\ Korrespondenzadresse \\ Dr. Felix Wagner \\ Dermatologiezentrum Neumünster, Kuhberg 43-45, \\ 24534 Neumünster, Deutschland \\ Felix.W.Wagner@gmail.com
}

\section{ZUSAMMENFASSUNG}

Präoperativ wird zur Vermeidung von Eingriffsverwechslungen die zu operierende Extremität mittels Farbmarkierungen detektiert. In der Phlebologie dient die Farbmarkierung darüber hinaus zum Auffinden der zu operierenden Varikose am Bein und gibt Hinweise darüber, welche Areale vor der Operation betäubt werden müssen. Das dabei verwendete Farbmaterial sollte der mehrmaligen gründlichen Desinfektion/Antisepsis der Haut des Operationsgebiets standhalten.

In der Vergangenheit wurde bisher nur selten von Kontaktekzemen ausgelöst durch Permanentmarker berichtet. Wir präsentieren einen klinisch sehr beeindruckenden Fall einer Kontaktallergie auf einen grünen Permanentmarker.

\section{ABSTRACT}

Preoperatively, the limb to be operated on is detected by means of colour markings in order to avoid mix-ups. In phlebology in particular, the colour markings also serve to locate the varicosis to be operated on the leg and indicate which areas need to be anaesthetised before the operation. The colour material used should be able to withstand repeated thorough disinfection/antisepsis of the skin of the area to be operated. Contact eczema caused by permanent markers has been reported very rarely in the past. We present a clinically very impressive case of contact allergy to a green permanent marker.

\section{Kasuistik}

Wir berichten von einer 74-jährigen Patientin, die ca. 26 Stunden nach Markierung durch einen grünen Permanentmarker (Edding ${ }^{\circledR}$ 3300, Edding International GmbH, Ahrensburg) ein Kontaktekzem Typ IV nach Coombs und Gell entwickelte. Anamnestisch bestanden in der Vorgeschichte keine weiteren Grunderkrankungen, nur eine Pflasterallergie war bekannt. Eine atopische Diathese wurde verneint.

Die zu behandelnden Krampfadern werden in unserem Venenkompetenzzentrum präoperativ beim stehenden Patienten mit einem grünen Permanentmarker angezeichnet. Dieses sogenannte „Mapping“ dient zum einen zur Erleichterung der Operation durch Vereinfachung des Auffindens der erkrankten Vene beim liegenden Patienten, zum anderen detektiert es den Bereich, der präoperativ mittels Tumeszenz-Lokalanästhesie betäubt werden muss. Sollte es bei der Setzung der Tumeszenz-Lokalanästhesie zu einer Tätowierung kommen, erscheint die grüne Farbe der natürlichen Venenzeichnung noch am ähnlichsten.

Die geplante Operation verlief ohne weitere Komplikationen erfolgreich. Es wurde eine inguinale Crossektomie links mit zusätzlichen Miniphlebektomien in Tumeszenz-Lokalanästhesie durchgeführt. 26 Stunden nach Markierung der zu operierenden Vene entwickelte die Patientin am noch zu operierenden rechten Bein, strickt der eingezeichneten grünen Markierungslinien, juckende, erythematöse Plaques sowie vereinzelt Papeln und Vesiculae auf erythematösem Grund ( $\triangleright$ Abb. $\mathbf{1 a}$ a, b).

Wir stellten die Diagnose einer allergischen Kontaktdermatitis. Die Patientin berichtete aufgrund des ausgeprägten klinischen Befundes über starken Juckreiz sowie hohen Leidensdruck. Daher begannen wir eine systemische Therapie initial mit $100 \mathrm{mg}$ Prednisolon per os ( $1 \mathrm{mg} / \mathrm{kg} \mathrm{KG}$ ). Diese wurde 2-tägig um $25 \mathrm{mg}$ redu- 

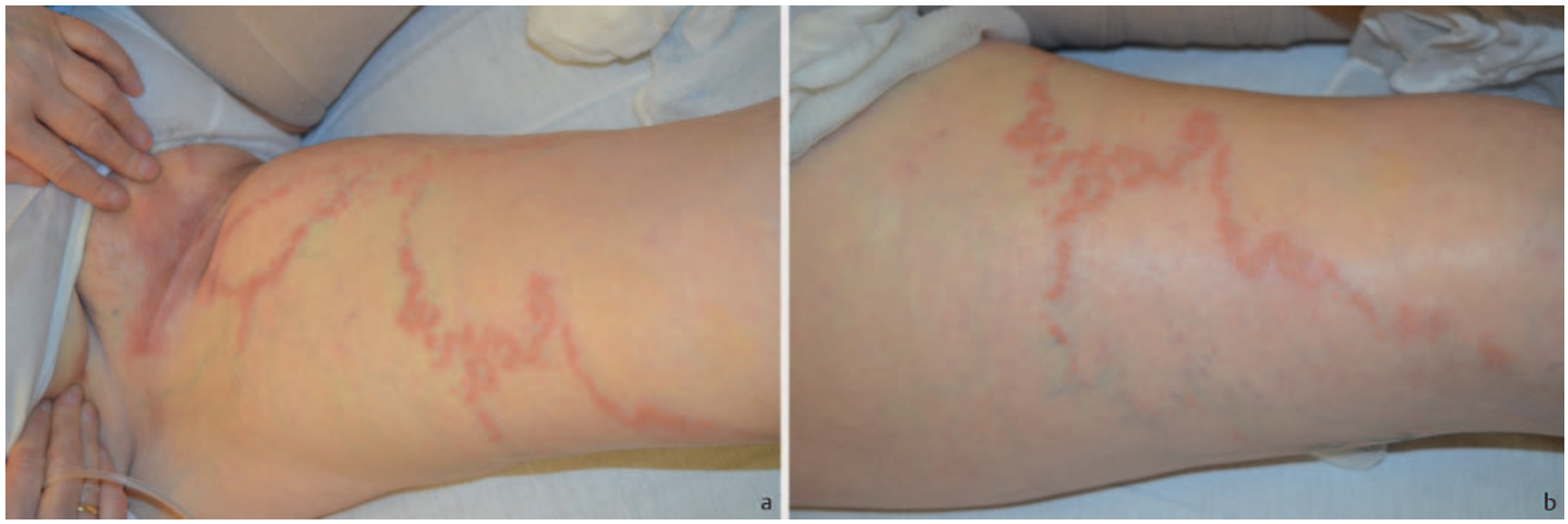

- Abb. 1 a, b Allergisches Kontaktekzem auf grünen Permanentmarker 26 Stunden nach Markierung mit juckenden, erythematösen Plaques sowie vereinzelt Papeln und Vesiculae auf erythematösem Grund.
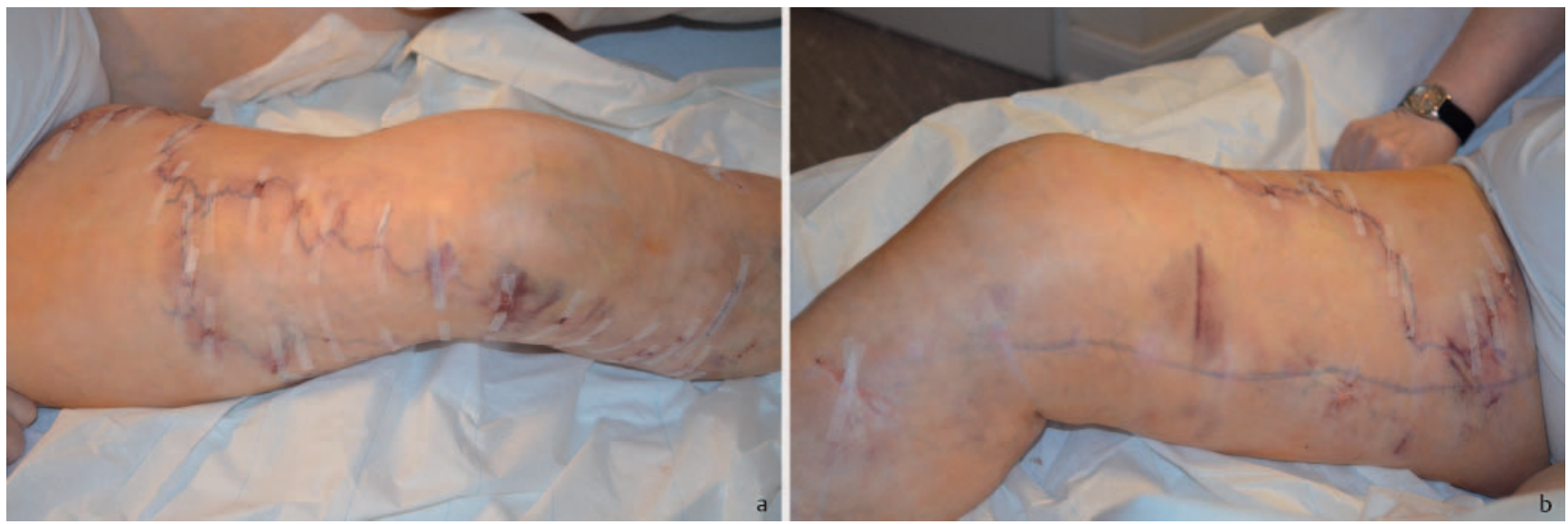

- Abb. 2 a, b Bein rechts 24 Stunden postoperativ nach Markierung durch einen schwarzen, zuvor getesteten Permanentmarkers.

ziert und konnte schließlich am 7. Tag abgesetzt werden. Begleitend führten wir eine topische Therapie mittels Diflucortolonvalerat durch. Da die Patientin auf die zugelassene Standarddosis von Cetirizindihydrochlorid $10 \mathrm{mg}$ Tbl. nicht ausreichend ansprach, erhöhten wir die Tagesdosis auf bis zu 2-mal täglich bei Bedarf (Off-Label-Use). Eine Höherdosierung von nicht sedierenden H1-Antihistaminika der 2. Generation auf bis zur 4-fachen Dosis wird bei Symptompersistenz bei der Therapie der chronischen Urtikaria empfohlen (ebenfalls Off-Label-Use) und ist uns bekannt. Innerhalb von 2 Wochen kam es hierunter zu einer deutlichen Besserung des Hautbildes und der Beschwerdesymptomatik. Zur weiteren Abklärung stellten wir die Patientin im Universitätsklinikum Hamburg-Eppendorf zur allergologischen Testung vor. Die Epikutantestung der DKG-Standardreihe sowie Lederund Textilfarben (ausgenommen Dispers Rot 11 und p-Phenylendiamin) zeigte eine 2 -fach positive Reaktion gegenüber Perubalsam sowie eine 3-fach positive Reaktion gegenüber dem verwendeten Permanentmarker der Firma Edding an Tag 2 und Tag 3 $(\triangleright$ Abb.3). Die restliche Epikutantestung war negativ.
Die Patientin berichtete uns über eine länger zurückliegende Operation mit Verwendung eines grünen Permanentmarkers. Hier sei es danach jedoch zu keiner Hautreaktion gekommen. Das innerhalb von 24 Stunden einsetzende Kontaktekzem sowie die 3-fach positive Testreaktion in der Epikutantestung sind hinweisend auf eine hochgradige Sensibilisierung. Bedauerlicherweise waren die Parafarbstoffe Dispers Rot 11 sowie p-Phenylendiamin bei der Epikutantestung laut Allergieambulanz nicht lieferbar. Eine erneute Epikutantestung im späteren Verlauf wurde seitens der Patientin abgelehnt.

Da die herstellende Firma keine näheren Informationen oder Materialproben aufgrund des Produktinhaltsgeheimnisses teilte, war eine weitere Aufschlüsselung mit Angaben der Zusammensetzung der im Farbstift enthaltenen Inhaltsstoffe nicht möglich. Die Operation an dem anderen Bein wurde mit einem schwarzem Permanentmarker (VISCOT SURGICAL SKIN MARKERS VISMARK'M fine REG 1400 Marker) durchgeführt, der präoperativ bereits mittels Epikutantestung sowie nach mehreren eigenständigen Selbstversuchen seitens der Patientin ohne Reaktion zur Anzeichnung freigegeben wurde $(\bullet \mathbf{A b b}$. 2a, b). 


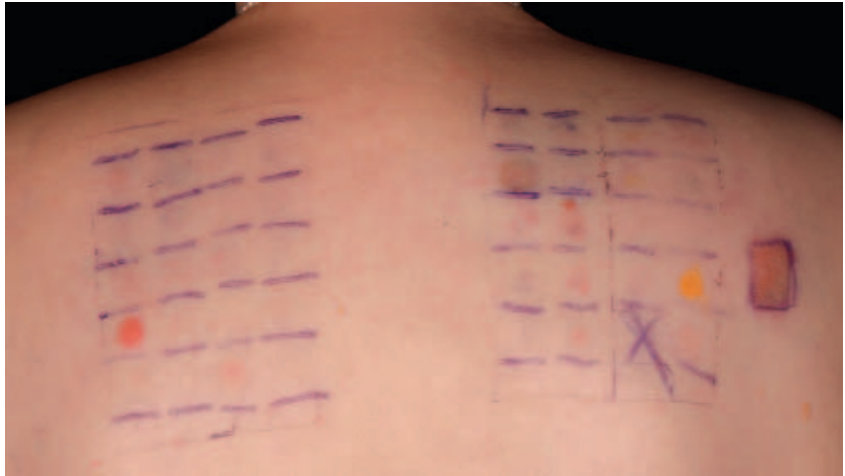

- Abb. 3 Erythem mit Infiltrat und konfluierenden Vesikeln. Epikutantestungsreaktionen extrem positiv (+++) am Tag 3 auf Perubalsam und Edding 3300 Permanentmarker grün. Quelle: Universitätsklinikum Hamburg-Eppendorf Allergologie.

\section{Diskussion}

Kontaktekzeme stellen eine der häufigsten Hauterkrankungen in Deutschland dar.

In Deutschland wird der von irgendeiner Form des Kontaktekzems betroffene Bevölkerungsanteil auf 15-20\% geschätzt. Die Lebenszeitprävalenz für das allergische Kontaktekzem wird auf etwa $15 \%$ geschätzt [12].

Allergische Kontaktekzeme sind durch Reizstoffe oder durch exogen einwirkende Allergene ausgelöste, akute, subakute oder auch chronische Entzündungsreaktionen. Das Ausmaß hängt vom Grad der Sensibilisierung sowie der Intensität des Allergenkontakts ab. Das allergische Kontaktekzem ist eine antigenspezifische, T-Zell-vermittelte, verzögerte Immunreaktion vom Typ IV (Gell u. Coombs, „verzögerter Typ") und kann zu einem klinisch polymorphen Bild führen. Die entzündlichen Hautveränderungen reichen von juckenden schuppenden Erythemen und Vesiculae und Plaques bis hin zu ausgeprägten Schwellungen mit Bullae oder gar Ulzerationen. Ebenfalls werden Streuphänomene vom primären Einwirkbereich des Allergens beobachtet. Diagnostisch ist neben einer gründlichen Anamnese und körperlichen Untersuchung vor allem der Epikutantest wichtig, um Typ-IV-Sensibilisierungen zu detektieren. Differenzialdiagnostisch sollte an ein kumulativ-toxisches Ekzem oder ein atopisches Ekzem gedacht werden. Aber auch die Psoriasis vulgaris oder das nummuläre Ekzem stellen wichtige Differenzialdiagnosen dar [9, 10, 12].

Sowohl die Compliance des Patienten als auch die Aufklärung des Arztes über die Erkrankung, das Ausschalten der diagnostisch ermittelten Noxen und die Schutz- und Pflegemaßnahmen sind für die langfristige Behandlung eines Kontaktekzems von entscheidender Bedeutung. Mittel der ersten Wahl zur symptomatischen topischen Therapie des allergischen Kontaktekzems sind Glukokortikoid-haltige Salben oder Cremes der Klasse II-III. Unterstützt wird diese durch eine konsequente längerfristige Basistherapie. Ist eine topische Therapie nicht wirksam oder undurchführbar kann bei besonders akuten, großflächigen oder therapierefraktären Fällen eine kurzzeitige systemische Therapie mit Glukokortikoiden über mehrere Tage bis 2 Wochen erfolgen [8].
Allergische Reaktionen in Form einer Kontaktdermatitis bzw. eines Kontaktekzem auf Inhaltsstoffe von Permanentmarkern sind äußerst selten, wurden jedoch in der Vergangenheit bereits von einigen wenigen Autoren beschrieben. Martin-Garcia et al. [1] zeigten in ihrem dokumentierten Fall ein nach 24 Stunden aufgetretenes allergisches Kontaktekzem ausgelöst durch einen blauen Permanentmarker ebenfalls der Marke Edding 3000 (Edding International GmbH, Ahrensburg) nach Liposuktion. Die nachfolgende Durchführung von Epikutantests in 3 verschiedenen Farben (rot, blau und schwarz) zeigten alle positive Testreaktion auf Abitol jeweils nach 48 und 96 Stunden. Abitol ist ein Gemisch aus verschiedenen Hydroabietylalkoholen und zählt im weitesten Sinne zur Gruppe der Klebstoffe. Es wurde hier erstmalig als auslösendes Allergen als eine Komponente des Permanentmarkers Edding 3000 beschrieben.

Ein ähnlicher Fall ereignete sich in der Klinik für plastische und rekonstruktive Chirurgie am Kantonspital Sankt Gallen. Matthias K. F. Spiegel et al. [2] berichteten von einer 52-jährigen Patientin, die einen Tag nach geplanter Mastektomie mit Sentinel-Lymphknotenexstirpation ein Kontaktekzem entwickelte. Das scharf begrenzte Erythem trat am ersten postoperativen Tag entlang der mit einem grünen Edding 3000 gezeichneten anatomischen Orientierungslinien auf. Im weiteren Verlauf entwickelten sich zusätzlich juckende Plaques und Vesiculae auf erythematösem Grund. Im Epikutantest zeigte sich an Tag 2 und 3 eine deutliche Sensibilisierung gegenüber Alkydharz. Alkydharze sind synthetische hydrophobe Polymere, die durch Kondensation mehrwertiger Alkohole mit mehrprotonigen Säuren unter Zusatz von Ölen bzw. Fettsäuren entstehen. Sie sind wichtige Inhaltsstoffe in vielen synthetischen Farben aufgrund ihrer Vielseitigkeit und niedrigen Kosten. Über Sensibilisierungen gegenüber Disperse Orange 3 und Solvent Yellow 146 (Orosal Yellow 4 GN) wurde in vergangenen Kasuistiken berichtet [3, 4]. Eine 62-jährige Patientin reagierte mit einem juckenden Kontaktekzem im Bereich der eingezeichneten Markierung auf denselben grünen Permanentmarker Edding 3000. Solvent Yellow 146 war hier jedoch das verantwortliche Allergen und zeigte sich im Epikutantest positiv.

Gemäß der World Health Organization sollte das präoperative Anzeichnen zur Vermeidung von Eingriffsverwechselung („Wrong Site Surfers“) mittels Permanentmarker erfolgen [5]. Eingriffsverwechslungen sind dadurch vermeidbar und können so großen individuellen Schaden abwenden. Es gibt jedoch aktuell immer noch keinen Konsens darüber, welcher Marker dazu genutzt werden sollte. Die meisten Permanentmarker beinhalten typischerweise keine länger anhaltenden toxischen Inhaltsstoffe. Anhand der Literatur wurden nur sehr selten allergische Reaktionen beschrieben. Permanentmarker sind nicht entwickelt worden für den direkten Kontakt auf der Haut. Eine Empfehlung der Hersteller von Permanentmarkern für den direkten Kontakt auf der Haut gibt es daher nicht. Spezielle Permanentmarker für die Haut sind im Handel erhältlich, welche bei einem Großteil der Operationen heute schon genutzt werden. Direkte Vorschriften zur ausschließlichen Nutzung dieser gibt es aufgrund der geringen Anzahl von Kontaktallergien auf handelsübliche Permanentmarker aktuell jedoch nicht. Bei der Verwendung von Permanentmarkern sollte 
daher auf eine hautfreundliche Testung geachtet werden, bzw. Patienten sollten über eine kontaktallergische Reaktion präoperativ aufgeklärt werden.

\section{Interessenkonflikt}

Die Autorinnen/Autoren geben an, dass kein Interessenkonflikt besteht.

\section{Literatur}

[1] Martin-Garcia C, Conde Salazar L, Gonzalez-Mendioca R et al. Contact dermatitis due to Edding 3000. Allergy 2004; 59: 235-236

[2] Spiegl MKF, Thraen AC, Hötzenecker W et al. Contact dermatitis caused by a green permanent marker. Contact Dermatitis 2018; 79 (4): 248-250. doi:10.1111/cod.13039. Epub 2018 May 30

[3] Komericki P, Kern T, Aberer W et al. Contact dermatitis from solvent yellow 146 in a permanent marker. Contact Dermatitis 2001; 44: 256

[4] Gonzalo-Garijo MA, Fernández-Durán DA, Pérez-Calderon R et al. Allergic contact dermatitis due to a temporary henna tattoo, a hair dye, and a marker pen. J Investig Allergol Clin Immunol 2008; 18: 226-227

[5] WHO Guidelines for Safe Surgery. Safe Surgery Saves Lives. Geneva, Switzerland: WHO. 2009; 2009

[6] Espasandin-Arias M, Vázquez-Osorio I, García-Martínez F] et al. Allergic contact dermatitis due to a surgical marker. Contact Dermatitis 2014; 71: $57-58$
[7] Puangpet P, White JM, White IR et al. Allergic contact dermatitis caused by a black permanent marker. Contact Dermatitis 2012; 67: 375-376

[8] Levin C, Maibach HI. An overview of the efficacy of topi- cal corticosteroids in experimental human nickel con- tact dermatitis. Contact Dermatitis 2000; 43: 317-321

[9] Brasch J, Becker D, Aberer W et al. Guideline contact dermatitis. S1-Guideline of the German Contact Allergy Group (DKG) of the German Dermatology 10 Leitlinie Kontaktekzem. Allergo J Int 2014; 23: 126 138. doi:10.1007/s40629-014-0013-5

[10] Peiser M, Tralau T, Heidler ] et al. Allergic contact dermatitis: epidemiology, molecular mechanisms, in vitro methods and regulatory aspects. Current knowledge assembled at an international workshop at BfR, Germany. Cell Mol Life Sci 2012; 69: 763-781

[11] Herausgeber: Tilo Biedermann, Werner Heppt, Harald Renz, et al. Allergologie 2016. Berlin Heidelberg: Springer Print ISBN: 978-3-642-372025, Electronic ISBN: 978-3-642-37203-2

[12] Belsito DV. The diagnostic evaluation, treatment, and prevention of allergic contact dermatitis in the new millenium. J Allergy Clin Immunol 2000; 105: 409-420

[13] Becker D. Allergic contact dermatitis. J Dtsch Dermatol Ges 2013; 11 : 607-622

[14] Klimek L, Vogelberg C, Werfel T. Weißbuch Allergie in Deutschland. Berlin, Heidelberg: Springer; 2019. ISBN: 978-3-89935-313-6 